\title{
CONTRIBUCIONES DE LA FILOSOFÍA DE LA CIENCIA A LA DIDÁCTICA DE LAS CIENCIAS
}

MELLADO, V. ${ }^{1}$ y CARRACEDO, D. ${ }^{2}$

1 Departamento de Didáctica de Ias Ciencias Experimentales y de las Matemáticas. Universidad de Extremadura. Badajoz.

2 Profesor de Filosofía de Enseñanza Secundaria. Badajox.

\section{SUMMARY}

In this article we show the importance of the philosophy science to found epistemologically science education. Later, we study the philosophical empiricist, rationalist schools and Popper, Lakatos, Kuhn, Laudan, Toulmin and Feyerabend. Finally, we establish analogies between the patterns of scientific knowledge in the studied schools and the patterns in scientific learning.

«Los conceptos físicos son creaciones libres del espíritu humano y no están, por más que parezca, únicamente determinados por el mundo exterior» (Einstein e Infeld, 1939, p. 34).

\section{INTRODUCCIÓN}

La ciencia forma parte inseparable de la cultura actual y es, probablemente, lo que más Ia diferencia de las anteriores (Bunge 1981). La concepción del mundo que tienen los hombres y mujeres de hoy está, en gran medida, determinada por el conocimiento científico y sus aplicaciones tecnológicas. Sin embargo, la imagen de la ciencia se ha visto a menudo simplificada y distorsionada al no considerarse los aspectos históricos y filosóficos de la misma.

El abandono de estos aspectos se constata en la escasa preocupación que tienen las carreras de ciencias básicas por la historia y la filosofía de la ciencia, lo que a menudo ha conducido a asumir implícitamente filosofías de la ciencia inmaduras e incontroladas (Bunge 1982).

La didáctica de las ciencias ha tenido un desarrollo progresivo en los últimos treinta años. Después de los proyectos de enseñanza por descubrimiento realizados en USA, Australia y Gran Bretaña y de los trabajos basados en la escuela de Piaget (Shayer y Adey 1984), destacamos los realizados en la última década sobre el constructivismo y el cambio conceptual (Driver 1986, 1988, Gil 1983, Hewson 1981, Hewson y Thorley 1989 , Posner et al. 1982, Novak 1988...). Durante estos años la didáctica de las ciencias se ha nutrido fundamentalmente de las propias disciplinas científicas básicas y de la psicología del aprendizaje.

Actualmente, la didáctica de las ciencias cuenta con un cuerpo teórico de conocimiento, se está configurando cada vez más como una disciplina específica y desde hace unos pocos años se están estudiando y debatiendo los fundamentos epistemológicos de la misma.

Desde la didáctica general (Marcelo 1990, 1992, Shulman 1986), se señala que el «conocimiento didáctico del contenido» es uno de los aspectos esenciales que debe conocer el profesor de ciencias. Este conocimiento didáctico del contenido sería distinto del conocimiento de la propia disciplina o del psicopedagógico general. A esta misma conclusión se ha llegado desde la didáctica de las ciencias (Furió et al. 1992, Gil 1992) cuando se indica que la didáctica de las ciencias tiene un campo 
específico de conocimiento y no es simplemente la suma de los contenidos de las asignaturas científicas básicas y de la psicopedagogía.

También en didáctica de las ciencias y hasta mediados de los 80 ha existido un alejamiento de la fílosofía de la ciencia (Dusch 1985). Sin embargo, en los últimos años, numerosos trabajos han considerado los aspectos fillosóficos a la hora de fundamentar epistemológicamente la didáctica de las ciencias (Gil 1983, 1986, Hodson 1986, 1988, Aliberas et al. 1989a, Nussbaum 1989, Clemison 1990, López Rupérez 1990, Burbules y Linn 1991, Cobb, et al. 1991, Dusch y Gitomer 1991...).

En nuestro caso consideramos la filosofía de la ciencia como un elemento esencial para el análisis y fundamentación de las disciplinas científicas. Además la filosofía de la ciencia puede ayudar a los profesores a explicitar sus puntos de vista sobre la construcción del conocí miento científico.

El fracaso de muchos cursos y programas sobre enseñanza de las ciencias tiene su origen en la ambigüedad que mantienen en sus posturas filosóficas, to que generalmente conduce a asumir implícitamente concepciones positivistas sobre la naturaleza de la ciencia, a pesar de haberse mostrado tales concepciones como inadecuadas para el aprendizaje científico (Hodson 1988).

En esta línea existen ya numerosas investigaciones que estudian, por métodos cuantitativos o cualitativos, las concepciones de los profesores sobre la naturaleza de la ciencia (Porlan 1988, Proper et al. 1988, Hewson y Hewson 1989, Eve y Dunn 1989, Kouladis y Ogborn 1989, Aguirre et al. 1990, Currais y Pérez 1992...).

Por otra parte, como señalaron Clark y Peterson (1986) desde la perspectiva del pensamiento del profesor, las concepciones del profesor tienen un reflejo e influencia en su actuación en el aula. También son numerosos los trabajos que, en contextos y niveles diferentes, estudian la relación entre las concepciones de los profesores sobre la naturaleza del conocimiento científico y su actuación en el aula al enseñar ciencias (Lederman 1986, Lederman y Zeidler 1987, Brickhouse 1990, Benson 1989, Tobin y Espinet 1989, Gallagher 1991, Cachapuz $1992 \ldots)$.

Este tema, creemos además que presenta una interesante actualidad en estos momentos en los que se está llevando a cabo en España una importantísima reforma, tanto en la organización del sistema educativo en niveles anteriores a la universidad, como en una completa reestructuración del currículo de las diferentes etapas, en cuya confección va a tener un papel protagonista el propio profesor, que deberá elaborar en equipo los proyectos curriculares de cada etapa.

En la aportación que realice a esta tarea quedará refle. jada sin duda la concepción última que el profesor posea sobre la fundamentación epistemológica de los distintos saberes, áreas y disciplinas, aspecto este que debe ser considerado como una de las fuentes en las que se sustenta y fundamenta el currículo.

\section{FILOSOFÍA DE LA CIENCIA}

Uno de los problemas presentes desde sus origenes en la historia de la filosofía ha sido la fundamentación del saber $o$, dicho en otras palabras, del conocimiento científico.

En la filosofía clásica, si exceptuamos a corrientes escépticas que van desde Pirron de Elis en la época helenística hasta diversos autores en épocas más modernas, se van decantando dos grandes corrientes que se explicitan en los siglos XVII y XVILl en las escuelas racionalista y empirista.

Los empiristas, que arrancan con la figura de Bacon y contirúan con Hobbes, Hume y Locke, ponen el acento en la justificación del conocimiento a partir de los datos suministrados por la experiencia sensible y tratan de establecer un método científico (inductivo y riguroso) apoyado en los datos de esta experiencia.

Esta corriente empirista ha tenido un importante papel en los primeros años de nuestro siglo en el llamado Círculo de Viena o el neopositivismo lógico (Kraft 1977). Destacan en el estudio de la fundamentación científica del conocimiento y en el rechazo de la metafísica o fílosofía tradicional como ciencia, poniendo el énfasis en el estudio y desarrollo de la nueva lógica matemática. El mismo Popper tuvo contactos con el Círculo, aunque más tarde manifestó su oposición.

La escuela racionalista, por el contrario, destaca la importancia que la razón y los conceptos creados por la mente tienen en el proceso de formación y fundamentación del conocimiento científico.

El creador principal de esta escuela en la época moderma es Descartes, aunque en el siglo siguiente surge la figura de Kant que con su idealismo transcendental intenta llegar a una superación o síntesis del enfrentamiento surgido entre las dos corrientes fílosoficas, estableciendo que si bien todo conocimiento científico tiene su origen en la experiencia sensible, tiene sin embargo que ser encuadrado en unas estructuras mentales transcendentales y a priori para que pueda ser realmente conocido y adquiera el rango de necesidad y universalidad que todo conocimiento científico debe poseer.

Ya bien entrado nuestro siglo se supera esta dicotomía entre las corrientes absolutistas, ya sean racionalistas o empiristas, y surge una posición constructivista para la que el conocimiento es una construcción de la inteligencia humana que va creando estructuras nuevas a partir de los conocimientos que se poseen.

Entre los autores que podemos de alguna manera encuadrar, aunque con muy distintos matices, en esta postura constructivista incluimos a Popper (falsacionismo) que puede ser considerado un filósofo de transición; Lakatos (metodología de investigación científica); Laudan (tradiciones de investigación); Toulmin (evolucionismo) y, finalmente, Kuhn (revolucionismo).

También en la época contemporanea existen algunas teorías relativistas de la filosofía de la ciencia que, como 
el anarquismo epistemológico de Feyerabend (1987, 1992), niegan la existencia de un conocimiento científíco universal. Parten de la base de que hay diferentes tipos de ciencia y de conocimiento científico, según las diferentes culturas y los diferentes entornos sociales en los que estas ciencias se desarrollen.

Uno de los criterios por el que podemos establecer una primera clasificación de las fílosofías de la ciencia estudiadas es el que se refiere a la posibilidad de evaluación de las teorías científicas.

Lakatos (1981) establece tres escuelas de pensamiento sobre este problema: a) el escepticismo, que piensa que no hay posibilidad de evaluar las teorías científicas; en este grupo incluye a Pirrón y a Feyerabend; $b$ ) demarcacionismo, que cree que existen criterios de demarcación para las teorías científicas y, además, que los criterios son universales; en este grupo se incluye a Popper y al mismo Lakatos; y c) el elitismo, formado por los que piensan que no hay criterios universales para evaluar las teorías científicas, en él se utilizarían criterios psicológicos, sociológicos e históricos y más que evaluar el producto científico se evaluaría a sus productores (la élite científica); en este grupo incluye a Kuhn y a Toulmin.

Porlan (1989) también establece una clasificación de las filosofías de la ciencia según su posibilidad de evaluación de las teorías científicas. En el caso de Kuhn considera que hay posibilidad de evaluar las teorías en los períodos de «ciencia normal», pero no en los períodos de «ciencia revolucionaria».

Basándonos en el esquema de Porlan, hemos elaborado la clasificación que se refleja en la figura I para un grupo de escuelas en filosofía de la ciencia a las que más adelante haremos referencia por su relación con teorías sobre el aprendizaje científico. El cuadro no pretende ser exclusivo y podría ampliarse con otros significativos filósofos de la ciencia que también podrian tomarse como referencia para fundamentar las teorías sobre el aprendizaje.

El falsacionismo de Popper (1983) considera que una teoría científica se rechaza por falsación cuando se encuentra un experimento crucial que la contradice. EI progreso científico se produciría por el repetido derrocamiento por falsación de las teorías y su reemplazo temporal por otras más satisfactorias. Es decir, en palabras de Popper, la ciencia crece por sucesivas conjeturas y refutaciones.

Lakatos, Laudan, Kuhn y Toulmin, en cambio, consideran que las teorías científicas son entes complejos que no pueden rechazarse por falsación y que no existen los experimentos cruciales.

Para Lakatos, todo programa de investigación científica tiene núcleos centrales resistentes al cambio, y a lo más que se llegaría con la falsación sería a rechazar hipótesis auxiliares que podrían fácilmente sustituirse sin alterar lo esencial. El progreso científico más bien se produciría por competencia entre programas, de tal forma que hay que considerar de forma simultáneamente las desventa- jas de lo viejo y las ventajas de lo nuevo. Se evalúan los progresos o degeneración de los programas de investigación.

\section{Figura 1}

Escuelas de pensamiento en filosofía de la ciencia según su planteamiento sobre la evaluación de las teorías científicas.

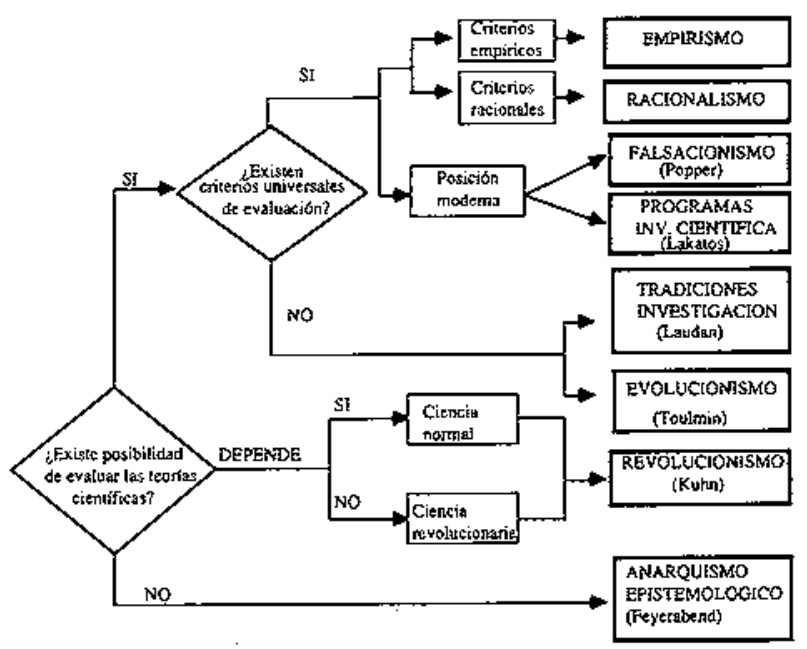

Kuhn (1971) subrayó la noción de paradigma como «conjunto de creencias, valores y técnicas compartidos por una comunidad científica». Existirían períodos de "ciencia normal» en los que domina un determinado paradigma. El cambio de paradigma se produciría en momentos de crisis, más por reconstrucción del campo -lo que implica nuevos fundamentos, métodos y aplicaciones-que por acumulación o ampliación del antiguo paradigma. Al partir de presupuestos distintos, los paradigmas sucesivos serían incomparables. El progreso científico existe en el sentido de que los nuevos paradigmas son más precisos y consistentes y tienen más capacidad de resolver los problemas.

Laudan (1986) defiende un modelo posibilista para el que la ciencia es una actividad cuyo fin es el de dar respuesta a una serie de problemas planteados. Establece el concepto de tradiciones de investigación (que incluye supuestos, metodologías, problemas y teorías) como semejante a los programas de investigación de Lakatos o a los paradigmas de Kuhn. La diferencia está en que el cambio científico se produce de una forma continua y la unidad de cambio sería la resolucion de problemas y no las tradiciones de investigación. El cambio de tradición de investigación (Estany 1990) se produciría cuando exista, además, un cambio ontológico y metodológico.

TouImin (1977) propone el concepto de ecología intelectual y establece una analogía entre la evolución biológica y la construcción del conocimiento científico. Las ideas científicas constituyen poblaciones conceptuales en desarrollo histórico y las teorías científicas cambiarían por evolución selectiva de las poblaciones conceptuales. Partiendo de los problemas no resueltos se produ- 
cirían unas exigencias intelectuales o unas prácticas específicas, que llevarían a una presión selectiva sobre las poblaciones conceptuales y, finalmente, a un desarrollo por innovación y selección (Porlan 1990).

\section{ANALOGÍAS ENTRE LA FILOSOFÍA DE LA CIENCIA Y EL APRENDIZAJE DE LAS CIENCIAS}

A continuación hacemos una revisión de las analogías que se han establecido en los últimos años entre la filosofía de la ciencia y el aprendizaje de las ciencias. Con estas analogías queremos mostrar que los modelos utilizados en didáctica de las ciencias también tienen raíces en la fílosofía de la ciencia. Sin embargo no queremos caer en un reduccionismo, ya que pensamos que la educación es un fenómeno extraordinariamente complejo y las simplificaciones pueden a veces aportar ignorancia (Morin 1992) si no se saben apreciar los aspectos globales.

En este sentido, el conocimiento de los aspectos filosóficos puede aportar visiones globales a la práctica del aula. Además, como señala Clemison (1990), ususcitando cuestiones sobre la estructura del conocimiento científico y cómo se desarrolla, podemos estar más seguros de que el currículo representa legítimamente el conocjmiento científico».

\section{Analogías entre el empirismo y el racionalismo y el aprendizaje de las ciencias}

Las posiciones positivistas empiristas consideran que el conocimiento es acumulativo y la verdad científica se descubre aplicando un procedimiento objetivo y riguroso que se conoce como el método científico (Fig. 2).

Esta posición ha sido duramente rechazada por la mayoría de las corrientes contemporáneas de filosofía de la ciencia, y es la base de dos concepciones de didáctica de las ciencias aparentemente distintas, pero con la misma raíz:

- Si la ciencia es un cuerpo de conocimiento, formado por hechos y teorías que se consideran verdaderos, entonces hay que transmitir a los estudiantes la verdad científica. Esto conduce a una enseñanza como transmisión de conocimientos elaborados, cuyo principal soporte es el libro de texto.

- Por otro lado, si el conocimiento se descubre aplicando el método científico, entonces hay que enseñar a los estuđiantes a realizar buenas observaciones, y a través de ellas y por inducción llegarán a descubrir las leyes de Ia naturaleza. Éste es el principio đe la enseñanza por descubrimiento autónomo tan en boga en los proyectos de hace treinta años.

Ambas tendencias, la enseñanza como transmisión de conocimientos elaborados y el aprendizaje por descubri-

Figura 2

Analogfas entre las escuelas empiristas y racionalistas con el aprendizaje de las ciencias.

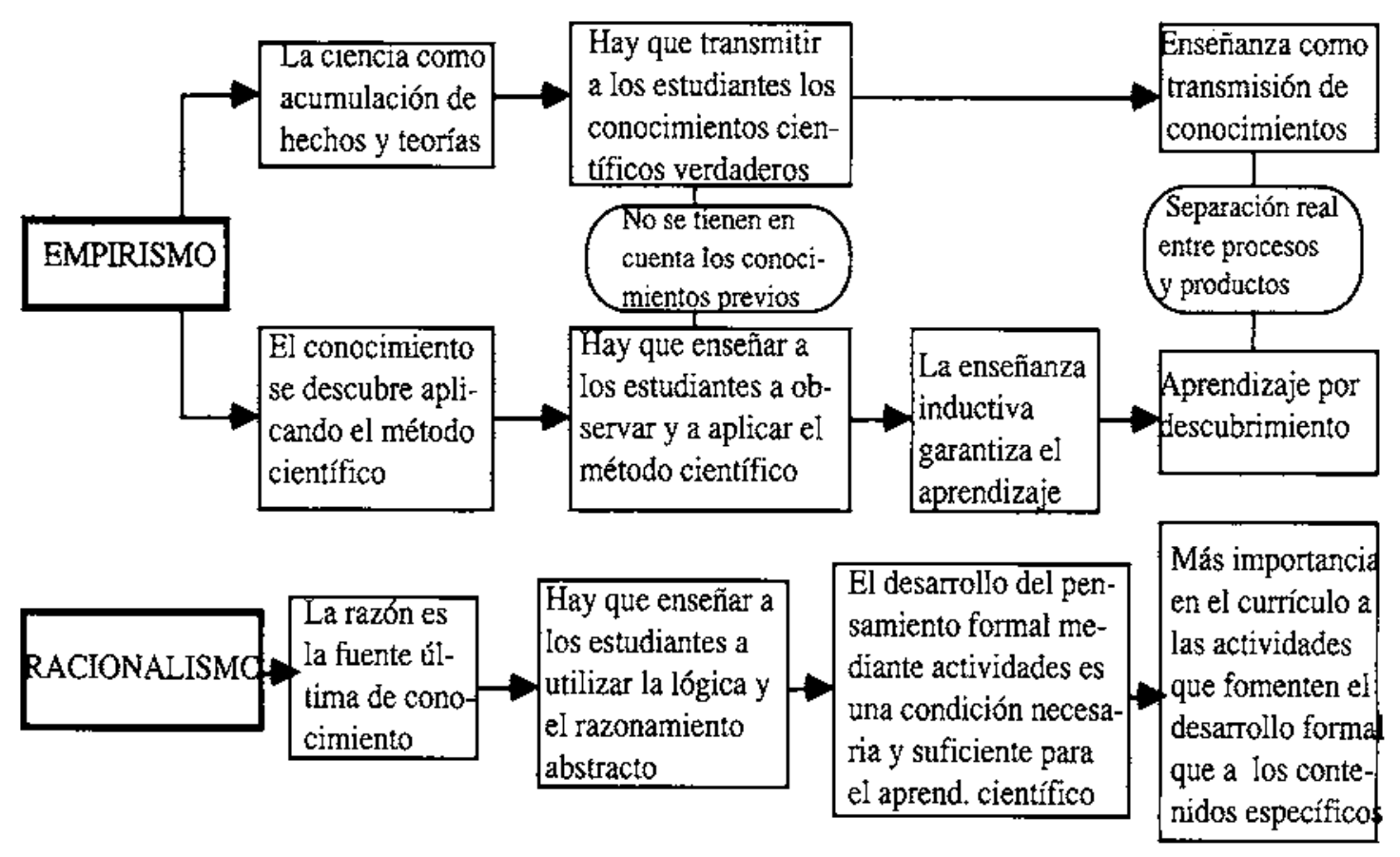


miento, han sido superadas por los modelos constructivistas; sin embargo, aún encuentran bastante aceptación como modelo didáctico implícito.

Brickhouse (1990) realizó una investigación cualitativa para determinar la relación entre las concepciones de los profesores sobre la naturaleza de la ciencia y su práctica en el aula. Sus resultados indican que las concepciones de Ios profesores sobre cómo se construye el conocimiento científico es consistente con sus creencias sobre cómo los estudiantes aprenden ciencia y, en consecuencia, con las metas instruccionales que se plantean los profesores.

Así, uno de los profesores estudiados por Brickhouse tenía concepciones positivistas empiristas. Esto se reflejaba en el aula en una separación real en el aprendizaje por los estudiantes de los procesos y los productos de la ciencia: los procesos los aprendían en el laboratorio aplicando el método científico, mientras que los productos (los hechos y teorías) los aprendían en el aula a través del libro de texto y de la explicación del profesor.
El aprendizaje por recepción significativa (Ausubel 1976) surgió como un rechazo al aprendizaje por descubrimiento autónomo. Supuso un avance respecto al anterior, ya que incidía en la importancia de las ideas previas de los alumnos en la integración del nuevo conocimiento así como en el papel de guía que el profesor đebe jugar en el proceso de aprendizaje; en este sentido introduce el término de descubrimiento guiado.

Sin embargo, desde el punto de vista epistemológico, persisten en el modelo de aprendizaje por recepción significativa aspectos inductivistas y empiristas. Las ideas alternativas de los alumnos serían «preconceptos» erróneos que hay que corregir (Aliberas et al. 1989b) y los conceptos se consideran externos al sujeto que tendría que «captarlos» (GiI 1992).

Para el racionalismo, la razón es la fuente última de conocimiento, e interpreta los hechos observables a través de teorías verdaderas a priori, construidas con una lógica universal.

Figura 3

Analogías entre las escuelas constructivistas de filosofía de la ciencia y el aprendizaje científico de los estudiantes.

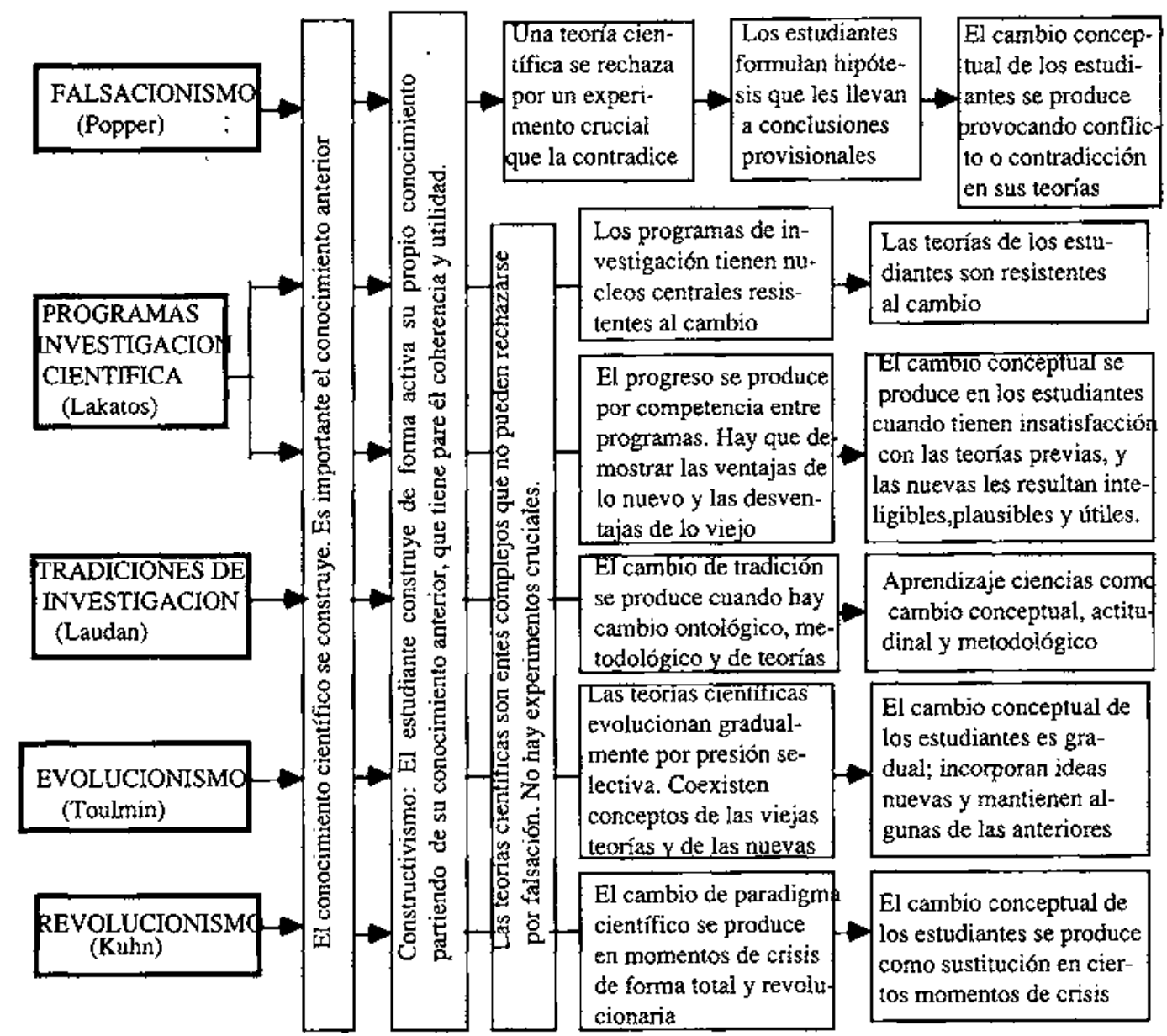


Según esta concepción, la mayoría de los errores de los estudiantes tendrán su causa en una incorrecta aplica. ción de la lógica y del razonamiento abstracto. En consecuencia, una meta instruccional es enseñar a los estudiantes a utilizar la lógica y el razonamiento abstracto.

Los aspectos estructurales de la teoría cognitiva de Piaget, pueden tener su fundamento en la tradición racionalista (Nussbaum 1989). Según Piaget el desarrollo condiciona el aprendizaje; el desarrollo se hace por etapas y en la última de ellas se aicanza el nivel del razonamiento abstracto o formal, que capacita para el aprendizaje científico. Este pensamiento formal se desarrollaría en todos los adolescentes por superación de la etapa anterior de las operaciones concretas; sería universal, y no estaría condicionado por las disciplinas específicas.

La consecuencia educativa, para los profesores que asumieron la concepción piagetiana, es que debían diseñar. se currículos que fomentaran el desarrollo de las operaciones formales. En estos currículos tendría más importancia el dominio del método a través de actividades para llegar a pensar formalmente que el dominio de contenidos disciplinares concretos, ya que, una vez conseguida la etapa de las operaciones formales, el alumno estaría en condiciones de entender cualquier contenido científico. Es decir, el desarrollo del pensamiento formal sería una condición necesaria, y casi suficiente, para el aprendiza* je científico.

Estos aspectos de la teoría de Piaget han sido rechazados por investigaciones posteriores (Pozo y Carretero 1987): el pensamiento formal no es universal, no todos sus esquemas se adquieren simultáneamente y el aprenđizaje depende de la disciplina científica y de las concepciones previas que tengan los estudiantes.

La teoría de Piaget, sin embargo, tiene otros aspectos que escapan a una simplificación racionalista de la misma, y que han contribuido decisivamente al conocimiento de los procesos de aprendizaje. Los aspectos funcionales de la teoría piagetiana (asimilación acomodación) tienen una base constructivista. Mientras para unos (Aliberas et al. 1989b), estos conceptos están próximos a las posiciones evolucionistas de Toulmin, para otros (Coob et al. 1991), Ia analogía se establecería con la noción de paradigma de Kuhn: la asimilación se realizaría dentro del paradigma dominante y la acomodación tendría su analogía con el cambio de paradigma. También hay que valorar el interés de la relación que ya establecía Piaget entre el desarrollo de las concepciones científicas del niño y la propia historia de la ciencia.

\section{Analogias entre las posiciones constructivistas de la ciencia y el aprendizaje de las ciencias}

Cinco de las escuelas filosóficas estudiadas, el falsacionismo, los programas de investigación científica, las tradiciones de investigación, el evolucionismo y el relativismo tienen una base constructivista. Consideran que el conocimiento científico es construido por la inteligencia humana, en un contexto generalmente social, tenien- do en cuenta el conocimiento existente, y por actos creativos en los que la teoría precede a la observación. Todas Ias teorías científicas tienen temporalmente una coherencia interna, y se corresponden con un cierto cuerpo de experiencias.

De forma análoga, la teoría constructivista del aprendizaje considera que el estudiante construye de forma activa su propio conocimiento, en el contexto social en el que se desenvuelve, y partiendo de su conocimiento anterior. Las teorías elaboradas por los estudiantes tienen también para ellos coherencia y utilidad, y se corresponden con las experiencias intuitivas que han tenido a lo largo de sus vidas.

A partir de aspectos comunes existen en las cinco escuelas filosóficas referidas notables diferencias en ia forma en que cambia el conocimiento científico y, en consecuencia, en la forma en que se produce el progreso científico. Estas diferencias podemos también observarlas, de forma análoga, en las diferentes tendencias constructivistas del aprendizaje.

En la figura 3 relacionamos de forma esquemática las posiciones de la filosofía de la ciencia sobre la construcción del conocimiento científico con las distintas corrientes didácticas sobre cómo se produce el aprendizaje científico (Mellado y Carracedo 1992).

No incluímos en el cuadro el relativismo de Feyerabend por tener unas características tan singulares. Algunos autores (Burbules y Linn 1991) consideran que el relativismo es antagónico no sólo con la didáctica de la ciencia, sino con la educación en general. Nosotros, sin embargo, creemos que hay un aspecto importante del relativismo que hay que considerar en didáctica de las ciencias y es el respeto hacia una serie de modelos que históricamente han supuesto avances en la enseñanza y aprendizaje de las ciencias en determinados contextos. El constructivismo se ha mostrado como un paradigma coherente y fundamentado para el aprendizaje de las ciencias, pero no puede considerarse como un paradigma dominante único, al estilo kuhniano, que excluya absolutamente a los demás. Como señala Shulman (1986), en educación, la coexistencia de escuelas divergentes de pensamiento lejos de ser una debilidad de desarrollo, puede ser más bien un estado natural y una muestra de madurez.

Por otras razones (Ray 1991), el transmitir un sólo punto de vista conduciría a un reduccionismo que la propia historia de la ciencia se ha encargado de refutar. Por ejemplo, el que el aprendizaje por descubrimiento se haya superado no quiere decir que haya que rechazarlo en todos sus términos. Hay que conocerlo y valorar la aportación positiva que en su momento significó la idea de aproximar el trabajo del aula al de la actividad científica (Gil 1992).

El paraielismo educativo con el faisacionismo de Popper sería que el cambio conceptual se produce en los estudiantes cuando se provoca contradicción entre sus teorías previas y la experiencia. En consecuencia, la meta instruccional sería encontrar experiencias que provoquen la contradicción. 
Burbules y Linn (1987), sin embargo, mantienen que la enseñanza por contradicción no es suficiente para inducir el cambio conceptual, ya que al cabo de un cierto tiempo los estudiantes vuelven a sus concepciones ingenuas.

La característica de la resistencia al cambio de Lakatos ha sido encontrada por numerosos autores en las teorías de los estudiantes. Nussbaum (1989) considera que existe analogía entre la filosofía de Lakatos y la teoría del cambio conceptual de Hewson (1981) y de Posner y otros (1982). El cambio conceptual se produciría en los estudiantes cuando tengan insatisfacción con las ideas previas y simultáneamente las nuevas ideas les resuiten inteligibles, plausibles y útiles. La estrategia didáctica comenzaría con una identificación de las ideas alternativas de los estudiantes, seguidamente se produciría un conflicto cognitivo por el uso de contraejemplos, y se irían introduciendo las nuevas ideas con oportunidades para aplicarlas en situaciones diferentes.

Dusch y Gitomer (1991) también consideran los modelos de Kuhn y Lakatos como la base epistemológica de Ia teoría del cambio conceptual señalada. Cuando el cambio conceptual de los estudiantes suponga una débii reestructuración de sus ideas, habría una analogía con los períodos de ciencia normal de Kuhn o, en el caso de Lakatos, cuando se vea afectado el cinturón auxiliar de los programas de investigación científica. En cambio, cuando el cambio conceptual supone una fuerte reestructuración de las ideas de los estudiantes, la analogía sería con los pexíodos de ciencia revolucionaria o de cambio de paradigma de Kuhn o, en el caso de Lakatos, cuando se vea afectado el núcieo central del programa.

Para Ray (1991), el movimiento de resolución de problemas en didáctica de las ciencias tendría su fundamento epistemológico en el concepto de Kuhn de ciencia normal. En estos períodos, los científicos resuelven los problemas planteados dentro del paradigma dominante.

También (Hodson 1988) encuentra analogías, siguiendo el modelo kuhniano, entre el cambio conceptual individual y el cambio conceptual que se produce en la comunidad científica.

Toulmin considera que Las teorías científicas evolucionan por presión selectiva de las poblaciones conceptuales. Nussbaun (1989) apoya esta tesis para el aprendizaje científico y cree que el cambio conceptual en los estudiantes es gradual, de tal forma que se van incorporando ideas nuevas pero se mantienen algunas de las anteriores. Porlan (1990) también defiende el modelo de cambio gradual y continuo de Toulmin como aplicable al conocimiento cotidiano tanto de los niños como de los adultos.

El aspecto más significativo de la filosofía de Laudan es que para que se produzca un cambio en una tradición de investigación no basta con un cambio de teorías, sino que tienen que cambiar además los fines y la metodolo- gía. Desde esta perspectiva Dusch y Gitomer (1991) critican el cambio conceptual de Posner, Strike, Hewson y Gertzog (1982), basado en los modelos de Lakatos y Kuhn, que supone de forma incorrecta que el cambio de las teorías produciría de forma simultánea cambios ontológicos y metodológicos. Consecuentemente proponen un modelo didáctico, siguiendo a Laudan, en el que el cambio metodológico juega un papel esencial.

Gil (1992) también critica el modelo de cambio conceptual ya que le parece una estrategia "perversa» explicitar las ideas de los alumnos para a continuación rechazarlas. Propone una estrategia didáctica que plantea el aprendizaje como una investigación a través del estudio de situaciones problemáticas abiertas de interés para los alumnos: «el aprenđizaje es concebido así no como un simple cambio conceptual, sino como un cambio a la vez conceptual, metodológico y actitudinal». Otro aspecto señalado por Gil y que ha sido considerado desde diversas perspectivas (Coob et al. 1991) es que, de la misma forma que la ciencia se construye de forma social, también en el aprendizaje hay que reforzar los aspectos colectivos, el trabajo en colaboración y el clima del aula.

\section{CONCLUSIONES}

Consideramos que la fílosofía de la ciencia puede contribuir a fundamentar epistemológicamente la điđáctica de las ciencias.

En este artículo hemos señalado algunas analogías que existen entre la construcción del conocimiento científico para algunas escuelas de filosofía de la ciencia y el aprendizaje científico en el aula por los estudiantes. Inevitabiemente el resultado es esquemático, ya que la complejidad y riqueza tanto de los modelos filosóficos como de los del aprendizaje no se ređucen a los aspectos considerados.

En estos momentos, la corriente que genéricamente denominamos constructivista y, más espećfficamente, el cambio conceptual y metodológico se han mostrado como teorias coherentes y fundamentadas para el aprendizaje de las ciencias. No obstante, la propia historia de las ciencias nos ha enseñado la provisionalidad de las teorías, por lo que es necesario, por una parte, seguir investigando sobre ello y, por otra, conocer y valorar las aportaciones que otras teorías han realizado sobre el aprendizaje de las ciencias.

Por último, el tema tiene grandes implicaciones didácticas para la formación de profesores de ciencias: si las concepciones epistemológicas de los profesores tienen influencia en su actuación en el aula y las concepciones de los profesores son a menudo implícitas y alternativas, también habrá que aplicar en la formación del profesorado estrategias de cambio conceptual y metodológico. 


\section{REFERENCIAS BIBLIOGRÁFICAS}

AGUIRRE, M., HAGGERTY,S. y LINDER, C., 1990. Studentteachers' conceptions of science teaching and learning: A case study in preservice science education, International Journal of Science Education, 12(4), pp. 381-390.

ALIBERAS, J., GUTIÉRREZ, R. y IZQUIERDO, M., 1989a. La didáctica de las ciencias: una empresa racional, Enseñanza de las Ciencias, 7(3), pp. 277-284.

ALIBERAS, J., GUTYÉRREZ, R, y IZQUIERDO, M., 1989b. Modelos de aprendizaje en la didáctica de las ciencias, Investigación en la Escuela, 9, pp. 17-25.

AUSUBEL, D.P., 1976. Psicología Educativa. Un punto de vista cognoscitivo. (Trillas: México).

BRICKHOUSE, N.W., 1990. Teachers' beliefs about the nature of science and their relationship to classroom practice, Journal of Teacher Education, 41(3), pp. 53-62.

BENSON, G., 1989. Epistemology and Science Curriculum, Journal of Curriculum Studies, 21(4), pp. 329-344.

BUNGE, M., 1981. La ciencia, su método y su filosofia. (Siglo $\mathrm{XX}$ : Buenos Aires)

BUNGE, M., 1982. Filosofía de la física. (Ariel: Barcelona).

BURBULES, N.C. y LINN, M.C., 1991. Science education and philosophy of science: congruence or contradiction?, International Journal of science Education, 13(3), pp. 227241.

CACHAPUZ, A., 1992. Filosofia daciencia e ensino da química: Repensar o papel do trabalho experimental. Comunicación al Congreso «Las Didácticas Específicas en la Formación del Profesorado», Santiago, julio de 1992.

CLARK, M. y PETERSON,P.L., 1986. Procesos de pensamiento de los docentes, en Wittrock, LaInvestigación en la Enseñanza, III. Profesores y Alumnos, pp. 444-539. (Paidós: Barcelona).

CLEMISON, A., 1990. Establishing an epistemological base for science teaching in the light of contemporary notions of the nature of science an of how children learn science, Journal of Research in Science Teaching, 27(5), pp. 429455.

COBB, P., WOOD, T. y YACKEL, E., 1991. Analogies from the Philosophy and Sociology of science for understanding classroom life, Science Education, 75(1), pp. 23-44.

CURRAIS, J. y PÉREZ, M., 1992. Epistemología y enseñanza de las ciencias. Implicaciones en la formación del profesorado. Comunicación al Congreso «Las Didácticas Específicas en la Formación del Profesorado». Santiago, julio de 1992.

DRIVER, R., 1986. Psicología cognoscitiva y esquemas conceptuales de los alumnos, Enseñanza de las Ciencias, $4(1)$, pp. 3-15.

DRIVER, R., 1988. Un enfoque constructivista para eI desarrollo del currículo en ciencias, Enseñanza de las Ciencias, $6(2)$, pp. $109-120$.

DUSCH, R.A., 1985. Science Education and Philosophy of science, twenty-five years of mutually exclusive development, School Science and Mathematic, 87(7), pp. 541-555.

DUSCH, R.A. y GITOMER, D.H., 1991. Epistemological perspectives on conceptual change: Implications foreducational practice, Journal of Research in science Teaching, 28(9), pp. 839-858.
ESTANY, A., 1990. Modelos de cambio cientifico. (Crítica: Barcelona).

EINSTEIN, A. y INFELD, L., 1939. La fisica aventura del pensamiento. (Losada: Buenos Aires).

EVE, R. y DUNN, D., 1989. High School Biology Teacher and pseudoscientific belief: passing it on?, The Skeptical Inquirer, 13 , pp. 260-263.

FEYERABEND, P., 1987, Contra el método. (Ariel: Barcetona).

FEYERABEND,P., 1992. Democratizar el pensamiento, El país, Suplemento «Ayer empezó el futuro», 29 de diciembre, p. 5.

FURIO, C., GIL, D., PESSOA DE CARVALHO, A.M. y SALCEDO, L.E., 1992. La formación inicial del profesorado de educación secundaria: papel de las didácticas especiales, Investigación en la Escuela, 16, pp. 7-21.

GALLAGHER, J.J., 1991. Prospective and practising secondary school science teachers' kowledge and beliefs about the philosophy of science, Science Education, 75 (1), pp. 121133.

GIL, D., 1983. Tres paradigmas básicos en la enseñanza de las ciencias, Enseñanza de las Ciencias, 1(1), 26-33.

GIL, D., 1986. La metodología científica y la enseñanza de las ciencias. Unas relaciones controvertidas, Enseñanza de las Ciencias, $4(2)$, pp. 111-121.

GIL, D., 1992. Contribución de la historia y filosofía de las ciencias a la transformación de la enseñanza de la ciencia, en actas History of the Physical-Mathematical sciences and the Teaching of Sciences. (European Physical Society: Madrid).

HEWSON, P.W., 1981. A conceptual change approach to learning science, European journal of Science Education, 3(4), pp. 383-396.

HEWSON, P.W. y HEWSON, M.G., 1988. An appropriate conception of teaching science: A view from studies of science learnig, Science Education, 72(5), pp. 596-614.

HEWSON, P.W. y THORKEY, N.R., 1989. The conditions of conceptual change in the classroom, International Journal of Science Education, 11, pp. 541-553.

HODSON, D., 1986. Philosophy of science and science education, Journal of Philosophy of Education, 20(2). Version espantola: 1988, Porlan, Garcia y Cañal, Constructivismo y enseñanza de las ciencias, pp. 5-22. (Díada: Sevilla).

HODSON, D., 1988. Toward a philosophically more valid science curriculum, Science Education, 72(1), pp. 19-40.

LAKATOS, I., 1981. Matemáticas, ciencia y epistemología. (Alianza Universidad: Madrid).

LAUDAN, L., 1986. El progreso y sus problemas. Hacia una teoria del progreso científico. (Ediciones Encuentro: Madrid).

KOULAIDIS, V. y OGBORN, J., 1989. Philosophy of Science: An empirical study of teachers' views, International Journal of science Education, 11 (2), pp. 173-184.

KRAFT, V., 1977. El Circulo de Viena. (Taurus: Madrid).

KUHN, $\Upsilon$., 1971. La estructura de las revoluciones cientificas. (Fondo de Cultura Económicą: Madrid).

LEDERMAN, N.G., 1986. Relating teaching behavior and classroom climate to changes in student's conceptions of the nature of science, Science Education, 70(1), pp. 3-19. 
LEDERMAN, N.G. y ZEIDLER, D.L., 1987. Science teachers' conceptions of the nature of science: Do they really influence teaching behaviors?, Science Education,71(5), pp. 721-734.

LÓPEZ RUPÉREZ, F., 1990. Epistemología y didáctica de las ciencias. Un análisis de segundo orden, Enseñanza de las Ciencias, 8(1), pp. 65-74.

MARCELO, C., 1990. Introducción a la formación del profesorado. Teoría y Métodos. (Universidad de Sevilla: Sevilla).

MARCELO, C., 1992. Cómo conocen los profesores la materia que enseñan. Algunas contribuciones de la investigación sobre conocimiento didáctico del contenido. Ponencia presentada al Congreso «Las didácticas específicas en la formación del profesorado». Santiago, julio de 1992.

MELLADO, V. y CARRACEDO, D., 1992. «Aproximación a la didáctica de las ciencias desde la filosofía de la ciencia». Comunicación al Congreso «Las Didácticas Específicas en la Formación del Profesorado». Santiago, Julio, 1992.

MORÍN, E., 1992. Entrevista reatizada por Javier Valenzuela para la revista Babelia, suplemento de El Pais, 18-7-1992.

NOVAK, J.D., 1988. El constructivismo humano: hacia la unidad en la elaboración de significados psicológicos y epistemológicos, en Portan, García y Cañal, Constructivismo y enseñanza de las ciencias. (Díada: Sevilla).

NUSSBAUM, J., 1989. Classroom conceptual change: philosophical perspectives, International Journal of Science Education, I1, pp. 530-540.

POPPER, K.R., 1983. Conjeturas y refutaciones. El desarrollo del pensamiento científica. (Paidós: Buenos Aires).

PORLAN, R., 1988. El pensamiento científico y pedagógico de maestros en formación, en Porlan, García y Cañal, Constructivismo y enseñanza de las ciencias, pp. 193-203. (Díada: Sevilla).
PORLAN, R, 1989. Teoría del conocimiento, teoria de la enseñanza y desarrollo profesional. Las concepciones epistemológicas de los profesores. Tesis doctoral. Sevilla.

PORLAN, R., 1990. Hacia una fundamentación epistemológica de la enseñanza, Investigación en la Escuela, 10, pp. 3-32.

POSNER, G.J., STRIKE, K.A., HEWSON, P.W. y GERTZOG, A., 1982. Acommodation of a scientific conception: toward a theory of conceptual change, Science Education, 66(2), pp. 211-277. Versión española: Porlan, García y Cañal, Constructivismo y enseñanza de las ciencias, pp. 91-114. (Díada: Sevilla).

POZO, J.I. y CARRETERO, M., 1987. DeI pensamiento formal a las concepciones esponáneas: ¿Qué cambia en la enseñanza de la ciencia?, Infancia y Aprendizaje, 8, pp. 35-52.

PROPER, H., MARVIN, F. e IVANY, W. y G., 1988. World view projected by science teacher: A study of classroom dialogue, Science Education, 72(5), pp. 574-560.

RAY, C., 1991. Breaking free from dogma: Philosophical prejudice in Science Education, Science Education, 75(1), pp. $87-93$.

SHAYER, M. y ADEY,P., 1984. La ciencia de enseñar ciencia. (Narcea SA: Madrid).

SHULMAN, L., 1986. Paradigmas y programas de investigación en el estudio de la enseñanza: una perspectiva contemporánea, en Wittrock, La investigación de la enseñanza, $I$. Enfoques, teorías y métodos. (Paidos: Barcelona).

TOBIN, K. y ESPINET, M., 1989. Impediments to change: applications of coaching in high school science teaching, Journal of Research in Science Teaching, 26(2), pp. $105-120$

TOULMIN,S., 1977.La comprensión humana:I.El usocolectivo y la evolución de los conceptos. (Alianza Universidad: Madrid), 
